\title{
Ageing Studies on the First Resistive-MicroMeGaS Quadruplet at GIF++
}

\section{Preliminary Results}

\author{
B. Alvarez Gonzalez ${ }^{1, a}$, M. Bianco ${ }^{1}$, E. Farina ${ }^{1,2}$, P. lengo ${ }^{1}$, F. Kuger ${ }^{1,3}$, T. Lin ${ }^{4}$, L. Longo ${ }^{5,6}$, G. Sekhniaidze ${ }^{6}$, \\ O. Sidiropoulou ${ }^{1,3}$, M. Schott ${ }^{4}$, C.Valderanis ${ }^{4}$, and J. Wotschack ${ }^{1}$ on behalf of the ATLAS Muon Collaboration \\ ${ }^{1}$ CERN \\ ${ }^{2}$ University of Pavia (IT) \\ ${ }^{3}$ Bayerische Julius Max. Universitaet Würzburg (DE) \\ ${ }^{4}$ Johannes-Gutenberg-Universitaet Mainz (DE) \\ ${ }^{5}$ University of Salento (IT) \\ ${ }^{6}$ INFN (IT)
}

\begin{abstract}
A resistive-MicroMeGaS quadruplet built at CERN has been installed at the new CERN Gamma Irradiation Facility $(\mathrm{GIF}++)$ with the aim of carrying out a long-term ageing study. Two smaller resistive bulkMicroMeGaS produced at the CERN PCB workshop have also been installed at GIF++ in order to provide a comparison of the ageing behavior with the MicroMeGaS quadruplet. We give an overview of the ongoing tests at $\mathrm{GIF}++$ in terms of particle rate, integrated charge and spatial resolution of the MicroMeGaS detectors.
\end{abstract}

\section{Introduction}

A resistive-MicroMeGaS quadruplet built at CERN, serving as prototype of the ATLAS [1] MicroMeGaS (MM) for the New Small Wheel upgrade of the Muon Spectrometer [2], has been installed at the new CERN Gamma Irradiation Facility $(\mathrm{GIF}++)$ with the aim of carrying out a long-term ageing study. This detector has four active layers about $0.3 \mathrm{~m}^{2}$ each equipped with 1024 read-out strips and sputtered resistive layer for spark protection [3]. Two smaller resistive bulk-MicroMeGaS, $10 \times 10 \mathrm{~cm}^{2}$, produced at the CERN PCB workshop have also been installed at $\mathrm{GIF}++$ in order to provide a comparison of the ageing behavior with the MicroMeGaS quadruplet.

The detectors are exposed to an intense gamma $(\gamma)$ irradiation of instantaneous flux of about $50 \mathrm{MHz} / \mathrm{cm}^{2}$ (depending on the attenuation filters) provided by the 13.9 $\mathrm{TBq}{ }^{137} \mathrm{Cs}$ source of $\mathrm{GIF}++$, corresponding to 10 times more than the highest expected counting rate at the High-Luminosity Large Hadron Collider (LHC). The resistive-MicroMeGaS chambers will be exposed during several months at GIF++ in order to accumulate an integrated charge up to $\sim 1 \mathrm{C} / \mathrm{cm}^{2}$ which corresponds with the highest expected flux in the NSW for about 25 years of HL-LHC operation.

An overview of the ongoing tests at GIF++ in terms of particle rate, integrated charge and spatial resolution of the MicroMeGaS detectors is given in this document.

\section{Gamma Irradiation Facility at CERN}

The new Gamma Irradiation Facility $[4,5]$ is located in the North Area of the CERN Super Proton Synchrotron (SPS) and it has been fully operational since March 2015. GIF++ is motivated by strong needs from the LHC detectors and accelerator communities in order to perform longterm ageing studies. It is a unique place where high energy charged particle beams (mainly muons) are combined with a flux of photons $(662 \mathrm{keV})$ from a $13.9 \mathrm{TBq}{ }^{137} \mathrm{Cs}$ source. A filter system permits attenuating the photon rate in several steps to reach attenuation factors of several orders of magnitude $\left(\sim 10^{4}-10^{5}\right)$.

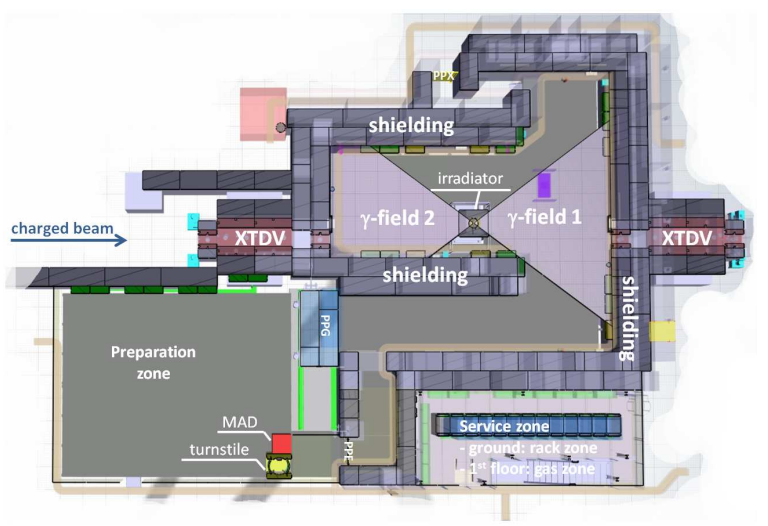

Figure 1. GIF++ bunker layout. The MicroMeGaS detectors are situated in the upstream area ( $\gamma$-field 2$)$ at $1 \mathrm{~m}$ from the source.

The high source activity produces a very intense background gamma field allowing to accumulate doses equiv-

\footnotetext{
a e-mail: balvarez@cern.ch
} 
alent to High Luminosity LHC (HL-LHC) experimental conditions in a reasonable time.

Figure 1 corresponds to the GIF++ area with the service and preparation zones and the inside of the bunker where the irradiator is placed. The MicroMeGaS detectors are situated in the upstream area $(\gamma$-field 2) at $1 \mathrm{~m}$ from the source (position U1) exposed to a $\gamma$ flux up to $64 \mathrm{MHz} / \mathrm{cm}^{2}$, see in Fig. 2.

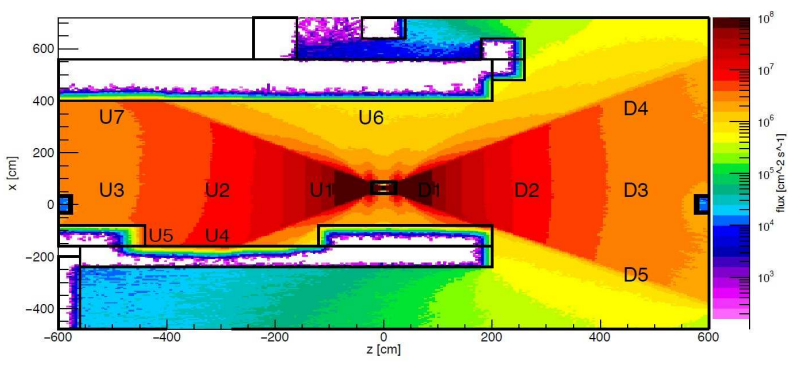

Figure 2. Total dose calculated from simulated flux. The values of the flux range from $10^{3}$ to $10^{8} \mathrm{~Hz} / \mathrm{cm}^{2}$. 'D' stands for positions downstream, and ' $U$ ' for positions upstream. The MicroMeGaS detectors are situated in the upstream area at $1 \mathrm{~m}$ from the source, position $\mathrm{U} 1(0.65 \mathrm{~m}, 2.0 \mathrm{~m},-1,1 \mathrm{~m})$.

\section{Experimental Set-up}

The detectors used for these studies are gaseous particle detectors detecting the traversing particles by amplifying the electrons that have been created by ionization in the gas volume. This gas volume is divided in two, the conversion gap and the amplification gap, by a metallic micro-mesh. Figure 3 shows the outline of the MM detectors with a $5 \mathrm{~mm}$ conversion gap and $128 \mu \mathrm{m}$ amplification gap.

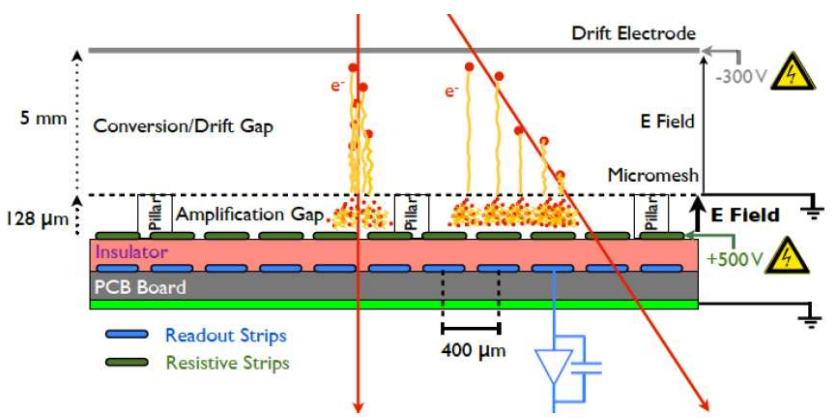

Figure 3. MicroMeGaS detector schema. HV numbers and dimensions correspond to the typical working conditions of ATLAS NSW MMs.

Ageing studies are mandatory to assess the capability of the detectors to the level of radiation at the HL-LHC. For the NSW MicroMeGaS, a very intense gamma source has been selected to perform the radiation studies.

Gamma radiation ionizes via three processes: photoelectric effect which is the dominant process for $\gamma$ with energies below $50 \mathrm{keV}$, Compton scattering, which is the dominant contribution in the photon energy range between 0.1 and $10 \mathrm{MeV}$ and is the main contribution at GIF++, and finally pair production which becomes important at energies over $5 \mathrm{MeV}$.

There are two types of MM detectors used in GIF++ for the studies described in this document. One corresponds to the prototype for the ATLAS New Small wheel (NSW) [2], MicroMeGaS Small Wheel (MMSW2), shown in Fig. 4. It is made of four active layers $\sim 0.3 \mathrm{~m}^{2}$, 4096 read-out strips and sputtered resistive layers, with mechanical floating mesh and strip pitch of $415 \mu \mathrm{m}$. It was exposed to the radiation source for one week during the muon beam period in August 2015.

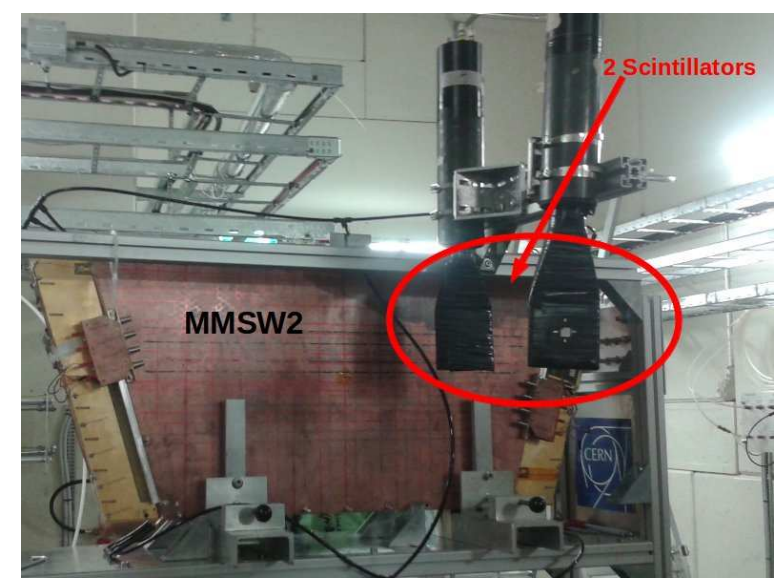

Figure 4. MMWS2 detector plus the two scintillators used for the trigger signal.

The other detectors are two resistive bulkMicroMeGaS chambers, with an active area of $10 \times 10$ $\mathrm{cm}^{2}$ and strip pitch of $400 \mu \mathrm{m}$, called $\mathrm{T} 5$ and T8, shown in Fig. 5. Those chambers have been exposed at GIF++ for more than 3 months and more detailed studies described in Sec. 6 have been performed.

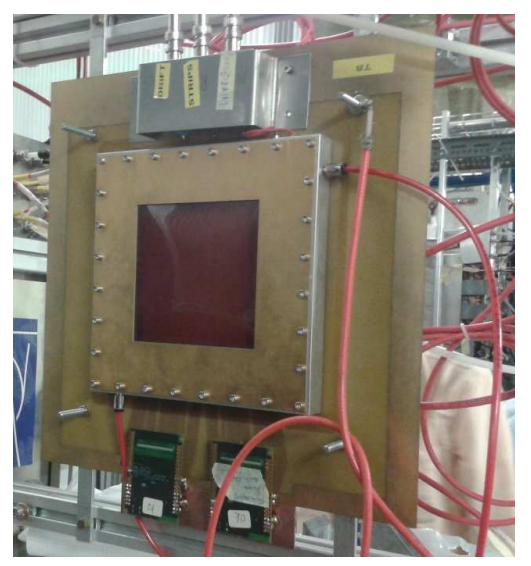

Figure 5. T8 in the frame during the GIF++ data-taking.

For the trigger signal during muon beam, two $10 \times 10 \mathrm{~cm}^{2}$ standard scintillators (highlighted in Fig. 4) were used in coincidence with a third scintillator located about $2 \mathrm{~m}$ away upstream. 


\section{Measurements}

The measurements are performed varying the amplification voltage and the attenuation filters. The attenuation factors used for our measurements range from 1 to $10^{4}$ in different steps; for instance we used the following values $\left[1,2.2,4.6,10, \ldots, 10^{4}\right]$. The amplification voltages used for the MMSW2 chamber go from 480 to $570 \mathrm{~V}$ in $10 \mathrm{~V}$ steps and for the bulk chambers from 420 to $540 \mathrm{~V}$ in $10 \mathrm{~V}$ steps as well. The voltage difference between the chambers is due to MMSW2 having a mechanical floating mesh.

As mentioned already above, there is the possibility of combining the source with a high energy muon beam which was used for some resolution studies presented in the following section.

The working conditions during the measurements were as follows:

- Gas type: $\mathrm{Ar} / \mathrm{CO}_{2}$ 93\%, 7\%

- Gas Flow: 5 1/h

- Amplification Gap: $128 \mu m$

- Amplification Field: $30-45 \mathrm{kV} / \mathrm{cm}$

- Drift Gap: $5 \mathrm{~mm}$

- Drift Field: $600 \mathrm{~V} / \mathrm{cm}$

- Operating Gain: $\sim 5 \times 10^{3}$

In total, more than 25 runs with $25 \mathrm{k}$ events each were collected and consequently analyzed.

\section{Results for MMSW2}

In Fig. 6 the current for the four layers of the MMSW2 chamber (L1, L2, L3 and L4) as a function of the amplification voltage is shown, in this case an attenuation factor of the order of 22 was used corresponding to an incoming photon flux of $3 \mathrm{MHz} / \mathrm{cm}^{2}$ on the MMSW2 detector. The curves show an exponential behaviour in all cases as expected.

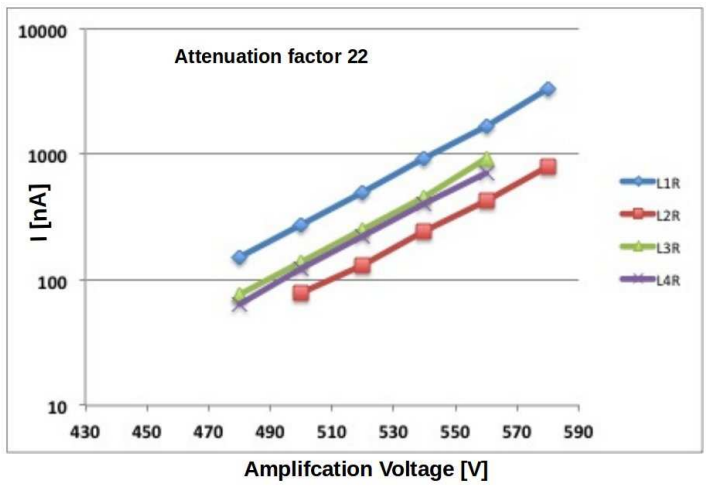

Figure 6. Current $[\mathrm{nA}]$ as a function of the amplification voltage [V] for the four layers of the MMSW2 chamber using an attenuation factor of the order of 22 corresponding to an incoming photon flux of $3 \mathrm{MHz} / \mathrm{cm}^{2}$.
To estimate the spatial resolution (for these preliminary results), the first step is to reconstruct the clusters for each event and for each layer of the MMSW2 chamber. Later a tracking algorithm is applied in order to define the track of a muon candidate using at least one cluster in all the layers. Then the distance between the cluster positions of a possible muon on L1 and L2 is calculated.

The distribution of the distance between the clusters on L1 and L2 is displayed in Fig. 7 for the maximum flux, no attenuation factor. The resolution is evaluated performing a gaussian fit to the distance distribution. Assuming that both layers have the same resolution, the final resolution is taken as the sigma of the gaussian fit divided by $\sqrt{2}$ leading to $\sim 100 \mu \mathrm{m}$ resolution. This resolution is evaluated by taking charge centroids in the clusters using perpendicular tracks. The results are not corrected by the effect of multiple scattering and beam divergence.

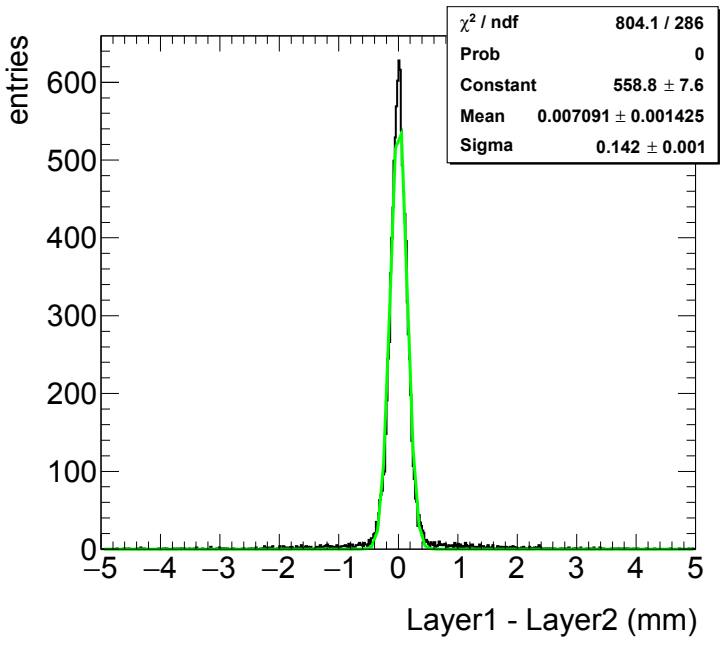

Figure 7. The distribution of the distance between the clusters on L1 and L2 for MMSW2 for the maximum flux, no attenuation factor. The fit is performed using a gaussian distribution function and the result of the fit is provided in the statistics box.

One can repeat the analysis for all attenuation points and study the resolution as a function of the flux as shown in Fig. 8. The resolution is flat up to the incoming flux of $\sim 10 \mathrm{MHz} / \mathrm{cm}^{2}$ which means that the algorithm used to select the muon candidates works successfully and provides the same resolution under high radiation conditions. The highest expected rate for the HL-LHC is about $15 \mathrm{kHz} / \mathrm{cm}^{2}$ [2]; taking into account the detector sensitivity to photons which is about $2 \times 10^{-3}$, a source rate of $\sim 7.5 \mathrm{MHz} / \mathrm{cm}^{2}$ would give the equivalent highest expected HL-HLC flux. The flux has been defined as the expected flux from simulation in the position of the chamber inside the GIF++ bunker. In addition, it has been proven that simulation agrees well with the measurements.

\section{Results for Bulk MicroMeGaS}

During the data-taking at GIF++, several runs were collected with several attenuation filters to estimate the particle rate. The particle rate in this case is the average number 


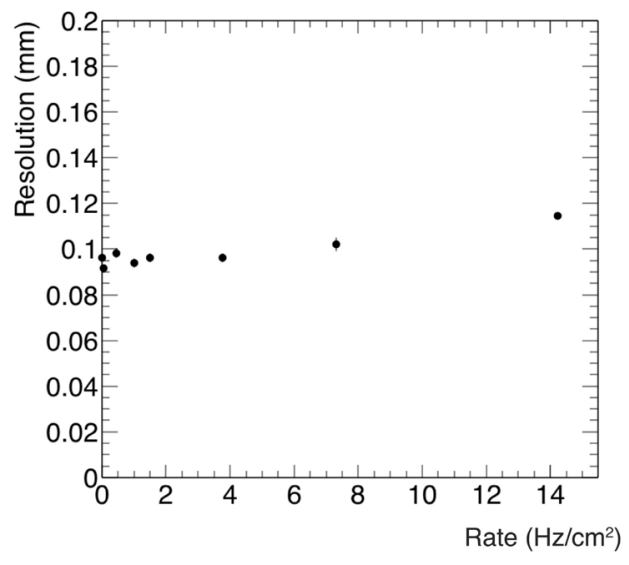

Figure 8. Resolution as a function of the incoming flux for L1 of MMSW2.

of photons (hits per event) counted in a time windows of $625 \mathrm{~ns}$ divided by the time window length.

Figure 9 shows the measured particle rate $\left(\mathrm{Hz} / \mathrm{cm}^{2}\right)$ as a function of the amplification voltage per attenuation factor. This measured rate includes the detector sensitivity to

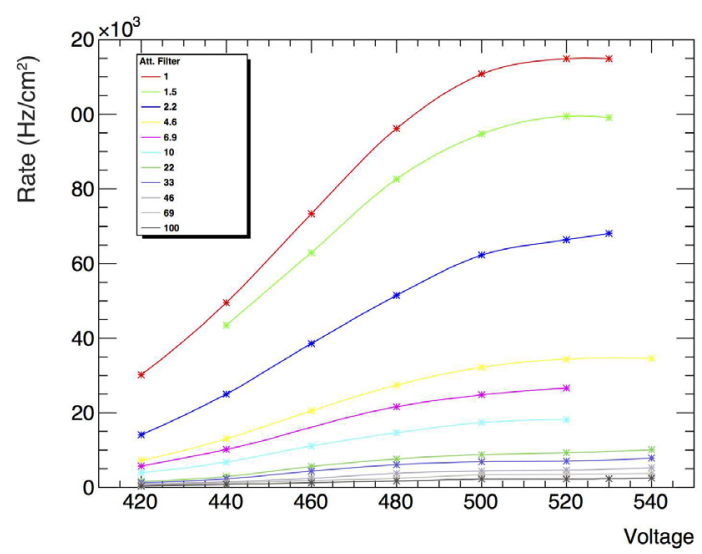

Figure 9. The measured particle rate as a function of the amplification voltage. The legend shows the different attenuation fa

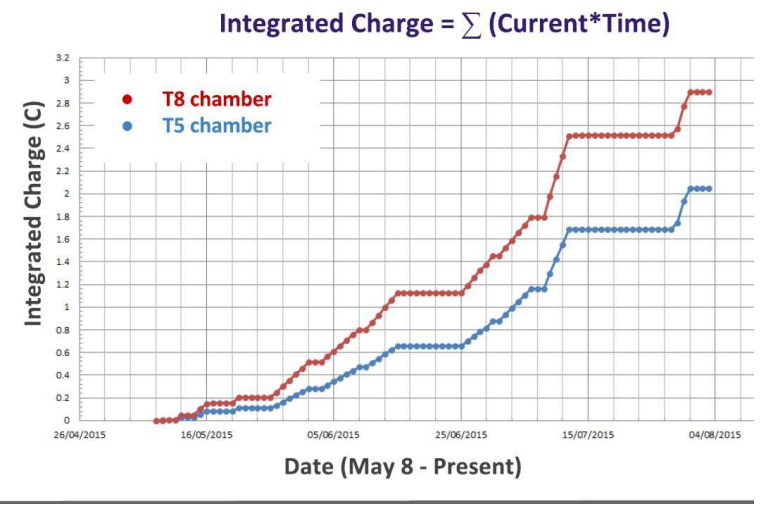

Figure 10. Integrated Charge as a function of time for $\mathrm{T} 5$ and T8. photons that is about $2 \times 10^{-3}$, the sensitivity accounts for the difference to the simulated flux values (per attenuation factor) and the measured particle rate.

Unlike the MMSW2 chamber, the T5 and T8 chambers have been exposed to the radiation in GIF++ for several months. Fig. 10 shows the integrated charge (C) from May to the beginning of August 2015; with a total of $\sim 3 \mathrm{C}$ $\left(0.03 \mathrm{C} / \mathrm{cm}^{2}\right)$ no degradation of the detector performance was observed.

\section{Prospects and Plans}

The plan is to expose of the resistive-MicroMeGaS chambers up to $\sim 1 \mathrm{C} / \mathrm{cm}^{2}$ in order to accumulate the highest expected flux in the NSW for about 25 years of HL-LHC operation and study the performance in this very high particle rate environment. The detector ageing evolution will be checked in terms of dark and amplification currents, efficiency and noise stability as a function of the integrated charge.

\section{References}

[1] ATLAS Collaboration, JINST 3 S08003 (2008).

[2] ATLAS collaboration, ATLAS-TDR-20-2013.

[3] P. Iengo et al., Construction of a large-size four plane MicroMeGaS detector. PoS (TIPP2014) 058.

[4] M.R. Jakel et al., CERN GIF++: A new irradiation facility to test large-area particle detectors for the highluminosity LHC program, PoS(TIPP2014)102.

[5] http://ph-dep-dt.web.cern.ch/irradiation-facilities/gif 